Abb. 1 Pupillenabflachung: Pupillenrandphänomene sind Ergebnisse von Kraftimpulsen beziehungsweise Impulsausfällen. Die betroffenen Abschnitte werden den Organen in den zugehörigen Sektoren zugeordnet.

\title{
Stress und Resilienz: Was die Iris erzählt
}

\author{
Die AUGENDIAGNOSE \\ kann entscheidende \\ Hinweise auf emotions- \\ bedingte Organbelas- \\ tungen liefern. \\ Hermann Biechele
}

KENNEN SIE DAS AUCH? Ein Patient kommt zum ersten Mal in die Praxis und berichtet von seinen Beschwerden und zahlreichen Besuchen bei Behandlern unterschiedlicher Fachrichtungen - ohne dass eine klare Diagnose gestellt werden konnte. Das macht ihn mut- und hilflos und stellt Sie vor eine Herausforderung. Wie gut, wenn Sie dann auf weitere Diagnosemöglichkeiten zurückgreifen können, zu denen auch die Augendiagnose gehört. Denn sie liefert uns spezielle Informationen über den Organismus, die in dieser Form mit keiner anderen Methode zu erhalten sind.

Merke: Im Gegensatz zur Irisdiagnose befasst sich die Augendiagnose neben der Iris auch mit dem Augenweiß, den Gefäßen und dem Augenumfeld (Wimpern, Brauen, Augenlider). 


\section{Genotyp und Phänotyp - Augenbefund und Somatismus}

Am Anfang steht die möglichst unvoreingenommene Befunderhebung per Irismikroskop aus dem Auge, gemäß dem Grundsatz: „Genotyp und Phänotyp zusammen ergeben die psychosomatische Union des Individuums“ [1, S. 12]. Damit erhält der kundige Therapeut eine Fülle an Informationen und Hinweisen auf eine individuelle, patientenzentrierte Therapie. Hierbei dauert die konzentrierte Befunderhebung gerade einmal zwei Minuten. Damit Augendiagnose aber nicht zum Reflexfeld für die Fantasie des Betrachters wird, sollte man ihre Möglichkeiten und Grenzen kennen.

\section{Phänomenologie, Empirie, Evidenz: Augenbefund als starker Marker}

Die Zeichen des Auges sind entweder genetisch, angeboren oder erworben. Wir erkennen diese Phänomene als Abweichungen von einer gedachten „Idealiris“ und geben ihnen eine entsprechende Bedeutung. In diesem Sinn ist Augendiagnose zuallererst Phänomenologie. In einem weiteren Schritt greifen wir auf die Erkenntnisse zurück, die wir zu den beobachteten Phänomenen gewonnen haben. Augendiagnose beruht also zu großen Teilen auf Empirie. In einem letzten Schritt ziehen wir dann entsprechend

\section{- INFORMATION}

\section{Was die Augendiagnose nicht leisten kann}

Die Augendiagnose kann keine klinische Organdiagnose liefern, weil wir im Auge kein Organ oder Gewebe des Körpers selbst erkennen. Wir sehen auch weder das Immunsystem mit seinen zellulären und humoralen Bestandteilen noch Hormone, Neurotransmitter oder elektrische Ladungen. Die einfache Zuordnung eines bestimmten augendiagnostischen Zeichens zu einer klinisch definierten Krankheit ist also nicht möglich.

\title{
KURZ GEFASST
}

\author{
1 Augendiagnose ist Phänomenologie, klinische Diagnosen \\ erschließen sich daraus nur empirisch. \\ 7 Strukturzeichen kennzeichnen Dispositionen und damit mögliche \\ Einbruchstellen. \\ Reflektorische Zeichen, Depositionszeichen und Pigmente kenn- \\ 3 zeichnen Diathesen und weisen auf typische pathophysiologische \\ Reaktionsmuster hin.
}

dem naturheilkundlichen Denkmodell therapeutische Schlussfolgerungen. Das ist Evidenz im besten Sinn: Sie gewährt Einsicht in Funktionsstörungen eines Systems beziehungsweise in systemübergreifende konsensuelle Zusammenhänge. Wir sehen die Iris mit ihrer individuellen Struktur: Was könnte zu dieser Zeichensetzung geführt haben? Welche Funktionsstörung oder Fehlsteuerung steckt dahinter, und was bedeutet das für den Patienten? Welche Krankheiten könnten aus einer solchen Konstellation entstehen?

\section{Systemische Zusammenhänge statt Kausalketten beschreiben}

Körperliche und psychische Störungen mit ihren wechselseitigen Einflüssen sind dabei als Einheit zu verstehen. $\mathrm{Ob}$ eine psychische Störung als Ursache für eine organische Erkrankung, zum Beispiel Bronchialasthma oder eine Ulkuskrankheit, infrage kommt, ist aus naturheilkundlicher und augendiagnostischer Sicht eher zweitrangig. Denn die Naturheilkunde denkt in systemischen Zusammenhängen statt einfachen Kausalketten. Etwas provokant könnte man fragen: Wird Kranksein wirklich erklärt, weil man die einzelnen Reaktionsschritte beschreiben kann? Geht es nicht vielmehr, wie Willy Hauser beschreibt, um die „Seinseinheit Mensch“?

\section{Depression: Eher Symptomen- komplex als Krankheit}

Ängste, Erregungszustände, melancholische Phasen oder Trauer sind emotionale Grundzustände des Lebens und kom- men in der täglichen Praxis häufig vor. Doch die Leitsymptome Freudlosigkeit, Traurigkeit, Mutlosigkeit, Antriebslosigkeit bis hin zur völligen Starre stellen sich nur vordergründig als scheinbar einheitliches und gut diagnostizierbares $\mathrm{Ge}$ schehen dar. Tatsächlich handelt es sich um einen umfangreichen Komplex aus psychisch-seelischen, neuro-vegetativen und hormonellen Wechselwirkungen. Deshalb spricht man folgerichtig vom „Depressiven Syndrom“. Unter diesem Begriff haben dann auch die körperlichen Symptome der Betroffenen ihren Platz, darunter Appetitlosigkeit, Übelkeit, Durchfall, Bauchschmerzen oder Verstopfung, ebenso Kopfschmerzen, Kreislaufsensationen, Störungen der Haut und Schleimhäute. Die nicht mehr ganz neue Disziplin der Psychoneuroendokrinologie versucht in diesem Sinn zu zeigen, wie letztlich doch alles mit allem zusammenhängt - eine ureigen naturheilkundliche Vorstellung.

\section{Pupillenphänome: Asymme- trisch, vergrößert, abgeflacht?}

Einige häufiger vorkommende augendiagnostische Phänomene liefern wichtige Erklärungen und Indikationen zu psychosomatischen Organbelastungen. So schwankt die Pupillengröße physiologisch - abhängig von Lichtverhältnissen, Lebensalter und vegetativem Grundtonus. Pupillenveränderungen können aber auch auf psychische und physische Störungen hinweisen. Daher betrachten wir die Pupille nach Josef Angerer als „Manometer der psychosomatischen Dynamik“ [1, S. 21]. 


\begin{abstract}
Merke: Die Pupillengröße weist auf den aktuell veränderlichen vegetativen Grundtonus hin: Eine Großpupille steht für Sympathikotonie, eine Kleinpupille für Parasympathikotonie.
\end{abstract}

Pupillenveränderungen zeigen sich zum Beispiel als Entrundungen, Abflachungen, Achsenverschiebungen oder Seitendivergenzen. Partielle Abflachungen stehen in Zusammenhang mit segmentalen Störungen der Wirbelsäulendynamik. Der Projektionsort entspricht dabei den zugehörigen Pupillenabschnitten. Bei allen Abflachungen ist auch nach Organzeichen im Irissektor zu fahnden. Bei organischen Störungen stellen wir hier häufig auch vegetative, emotionale und psychische Einflüsse fest.

\section{Pupillenspiel als Gradmesser vegetativer Aktivität}

Das dynamische Spiel der Pupille verdeutlicht, wie es um die vegetative Stimmung und die Nervenfunktion steht. Ein lebhaftes Pupillenspiel weist auf ein überreiztes Nervensystem hin. Eine erhöhte oder reduzierte Schwingungsbreite verrät hypersensible beziehungsweise hyposensible Zustände im Reizvermittlungssystem. Diese können ihren Ausdruck in psychischer Übererregbarkeit einerseits oder Erschöpfung und Depression andererseits finden. Josef Angerer schreibt in Bezug auf diese komplexen Zusammenhänge: „An der Mechanik des Pupillenspiels beteiligen sich nicht nur alle Nervenarten, sondern auch alle Energieströme der psychosomatischen Einheit. Vielleicht ist daher hier eine bildhafte Energieschau gegeben." [1, S. 24]

\section{„Die Ahnung von der Einheit alles}

\section{Lebendigen scheint sich auch hier}

\section{zu verdichten."}

Josef Angerer über das Pupillenspiel

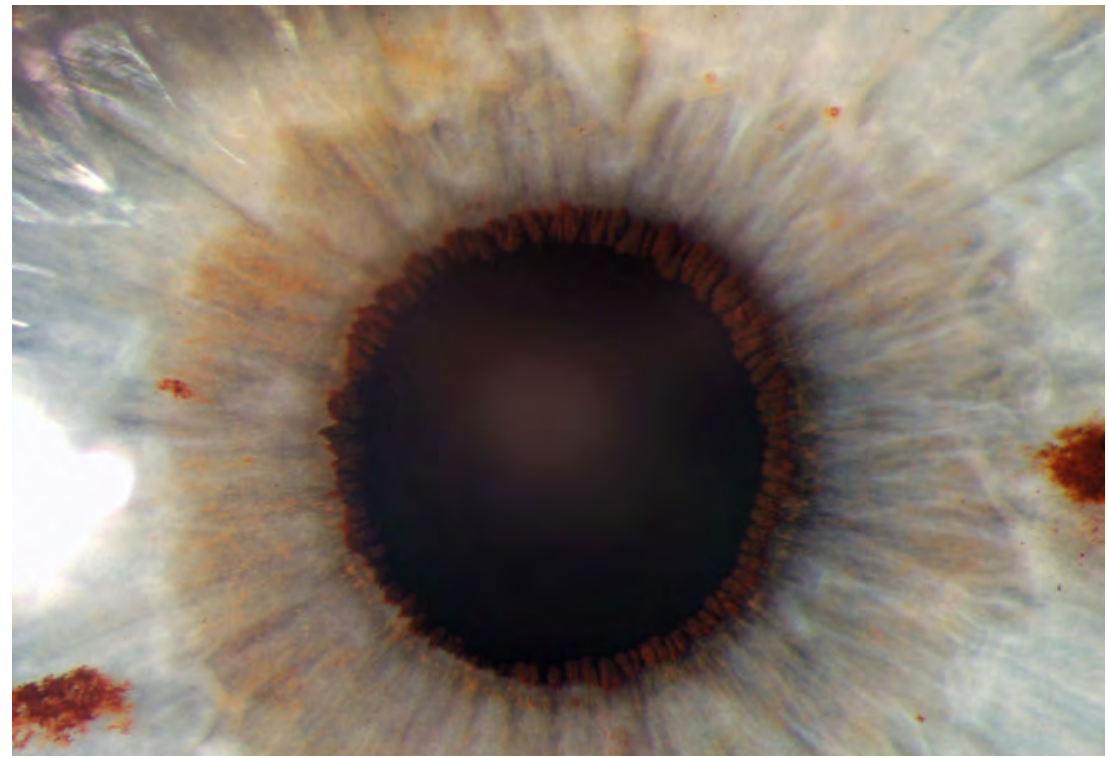

Abb. 2 Eine abschnittweise Verbreiterung des Pupillensaums, bei der die „Zähnchen“ wie Ziegelsteine aufeinanderliegen, ist ein relativ zuverlässiges Zeichen für nervöse Herzstörungen auf dem Boden eines allgemein schwachen und spasmophilen Nervensystems.

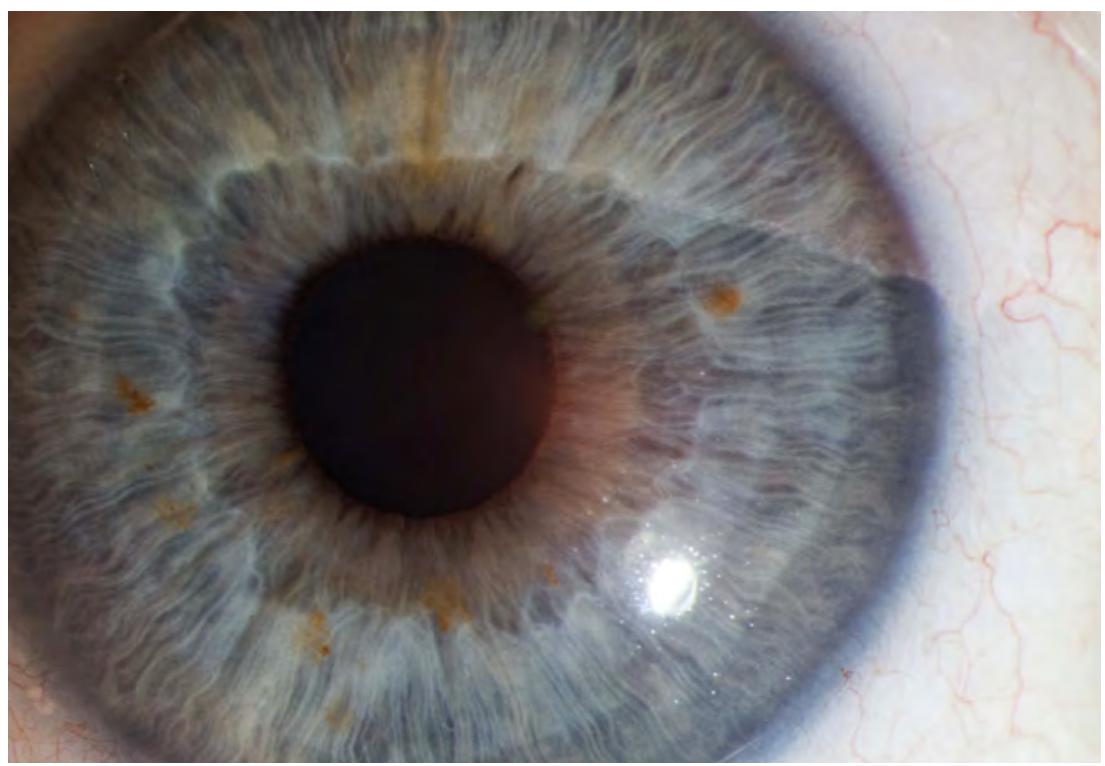

Abb. 3 Abflachungen im oberen Bereich der Iriskrause weisen neben Störungen des Colon transversum auch auf Psychoneurosen und depressive Tendenzen hin.

Pupillensaum als cerebrospinales Reflexfeld

Der Pupillensaum ist die sichtbare Umschlagstelle des Pigmentblatts der Iris. Er ist als Teil der Retina ein vorgeschobener Hirnteil und stellt in diesem Sinn ein cerebrospinales Reflexfeld dar. Die Form des Pupillensaums (dünn, breit, zart, grob) sagt etwas über die psychische Dynamik aus.
Merke: Der Pupillensaum ist ein anatomisches Substrat, gebildet von der Pigmentschicht. Mit Pupillenrand ist die Konfiguration der Pupille gemeint.

So weist ein dünner Pupillensaum auf eine erniedrigte spinale Reflexerregbarkeit mit verringerter Sensibilität hin. Vorzeitige Ermüdung und Adynamie sind die 


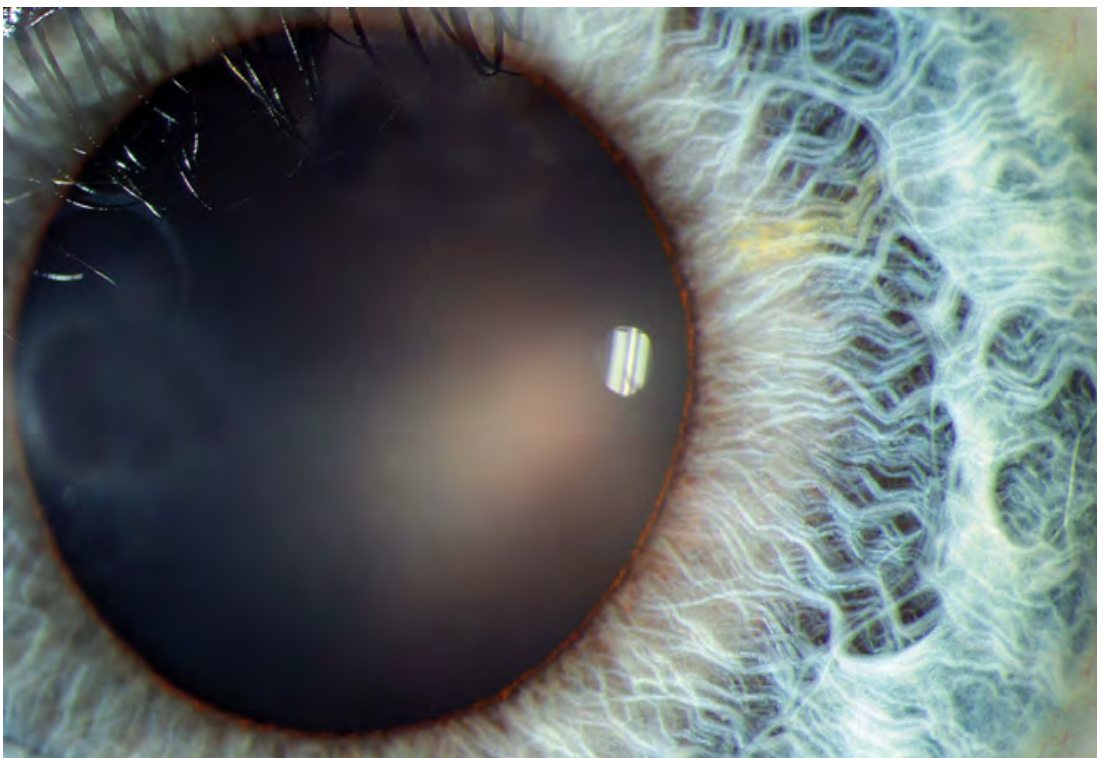

Abb. 4 Die partiale Verdoppelung des Krausenfadens entspricht einem labilen Nervensystem. Nervöse Überreizbarkeit und Organreaktionen, Tendenz zu Depressionen, Psychosen oder Witterungsneurosen können auftreten. Die Organe in den betroffenen Sektoren erfordern eine erhöhte Aufmerksamkeit.

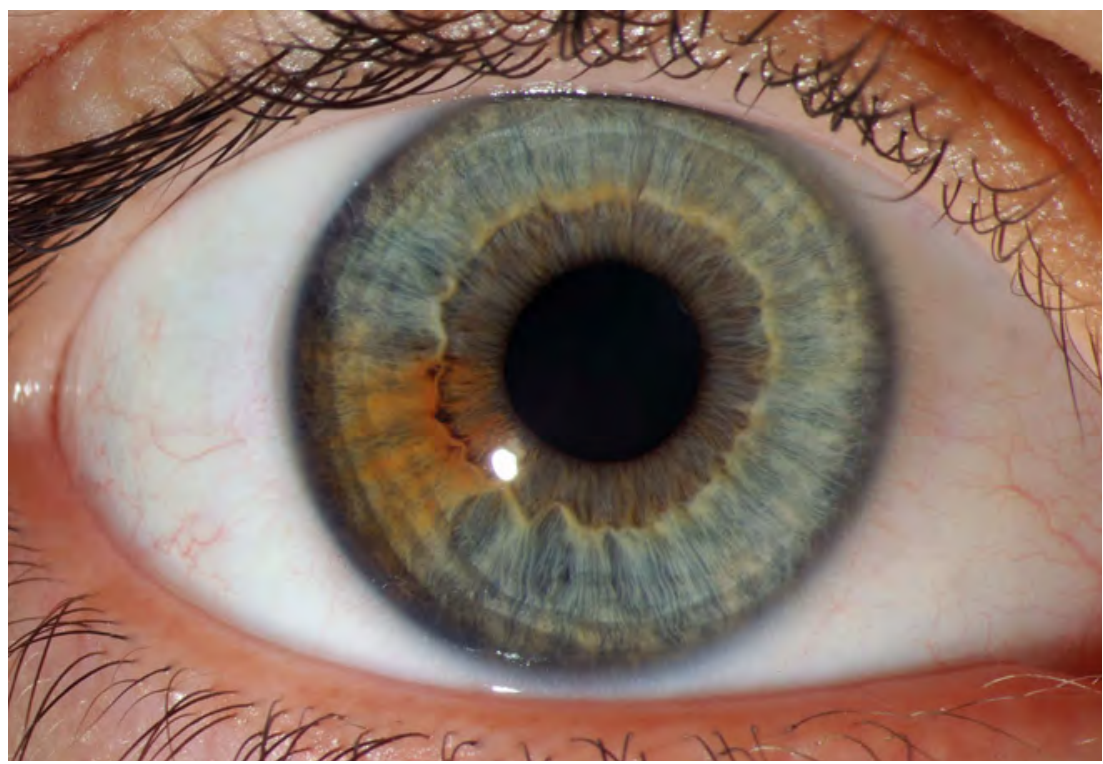

Abb. 5 Die sektorale Heterochromie wird von Schnabel [8] mit nervalen Funktionsstörungen in Verbindung gebracht. Je nach Lokalisation zeigen sich zum Beispiel Schilddrüsen-, Herz- oder Galleflussstörungen.

Folge. Die Struktur des Pupillensaums, vor allem der lokale Abbau, spricht für eine reduzierte physische Dynamik und Lebenskraft, wie sie typisch für den alternden Organismus ist. Die Farbe (hell, dunkel) gibt Auskunft über die Reaktionsfähigkeit auf Reize. Diese bezieht sich auf die Erfolgsorgane, die im betroffenen Sektor liegen. Auf diese Weise gilt der Pupillensaum nach Hauser als Repräsentant des Netzwerks „sympathisch-parasympathisch-enterales Nervensystem“.

\section{Iriskrause und Krausenzone: Zeichen für den vegetativen Grundtonus}

Die fadenartige Iriskrause unterteilt die Iris in die pupillenseitige innere Krau- senzone und die äußere Ziliarzone. Ihre Anlage ist genetisch vorgegeben und damit konstitutionell. Durch ihre Lokalisation in der Iris bestimmt sie die Größe der Krausenzone und zeigt damit den vegetativen Grundtonus an - insbesondere die Balance von Sympathikus-Parasympathikus und des enteralen Nervensystems. Die Architektur der Krausenzone (eng oder weit, lokale Aussackung oder Eindrückung der Iriskrause) gibt dazu wichtige Hinweise. Ein partiell gerader Krausenverlauf signalisiert Einschränkungen der vegetativen Steuerung bis hin zur Regulationsstarre in den betroffenen Abschnitten.

\section{Merke: Die Architektur der Krau- senzone liefert Informationen über den konstitutionell festgeleg- ten, vegetativen Grundtonus. Eine weite Krausenzone spricht für konstitutionelle Parasympathiko- tonie, eine enge Krausenzone für konstitutionelle Sympathikotonie. Der aktuelle Zustand wird unter anderem aus der Pupillengröße und dem Pupillenspiel erkennbar.}

In der Struktur der Iriskrause spiegelt sich auch die neurovegetative Reaktionsbereitschaft. Eine dünne, aufgefaserte, unterbrochene oder fehlende Iriskrause ist ein Zeichen für neurovegetative Labilität in Bezug auf Reizwahrnehmung (Sensibilität) und Reizbeantwortung (Irritabilität). Eine verdickte Iriskrause deutet in diesem Sinn auf eine Reaktionsschwäche des vegetativen Nervensystems hin.

\section{Die Krausenzone als magisches Auge der Nutritionsdynamik}

Nicht selten finden sich im Bereich der Krausenzone Veränderungen der Faserspannung. Bei nachlassendem Tonus sinkt die Zone schüsselförmig ein. Bei übersteigertem Tonus wölbt sie sich halbkugelartig nach vorne. Diese als „Trichterkrause“ beziehungsweise „Vorgewölbte Krause“ bezeichneten Phäno- 
mene signalisieren intestinale und psychische Korrelate, insbesondere seelische Aufnahmebereitschaft und Isolierung.

$\mathrm{Zu}$ achten ist auch auf eine sektorale Heterochromie (Farbabweichung). Diese kann auf die Krausenzone beschränkt sein und weist dann auf trophische Störungen im Verdauungstrakt hin. Erstreckt sie sich jedoch über das gesamte Irisareal von der Pupille bis zum Ziliarrand, ist mit tiefgreifenden psychischen Belastungen zu rechnen. Die Farbe des Pigments ist dabei ein zusätzlicher Hinweis auf den Entstehungsmodus, wobei Josef Angerer immer auf die Beteiligung des Hormonsystems verwies. Immerhin wissen wir heute, dass die Neurotransmitter Serotonin beziehungsweise Noradrenalin und Dopamin eine (entscheidende?) Rolle beim depressiven Geschehen spielen, was sich in der sogenannten „Serotonin-Hypothese der Depression“ niedergeschlagen hat.

Eva Flink liefert im Zusammenhang mit der Farbgebung in der Iris einen weiteren diagnostischen Beitrag: Pigmente im Herzfeld kennzeichnen demnach den „Grübler mit Neigung zu Depressionen“.

\section{Lakunen: Genetische „Schwächezeichen“}

Die Iris besteht vereinfacht gesprochen aus drei Schichten: vordere Grenzschicht, Faserschicht und Pigmentschicht. Sie bestimmen das Aussehen der Iris. Lakunen entstehen durch Lückenbildung in der schleierartigen vorderen Grenzschicht und sind ein Hinweis auf genetisch fixierte Schwachstellen. Gehäuft auftretende Lakunen rings um die Iriskrause sind das Kennzeichen der glandulär schwachen Disposition (Josef Deck) beziehungsweise endokrin-vegetativen Konstitution (Joachim Broy) im Sinne einer Anpassungsschwäche des endokrin-vegetativen Syndroms. Sie kann sich unter anderem als endokrine Störung, zum Beispiel eine Störung der Schilddrüse, juvenile Fettsucht oder Entwicklungshemmung auswirken. Aber auch neurovegetative Fehlleistungen, wie das Asthma juvenile, ein Leistungsknick der „mittleren Jahre“, An-

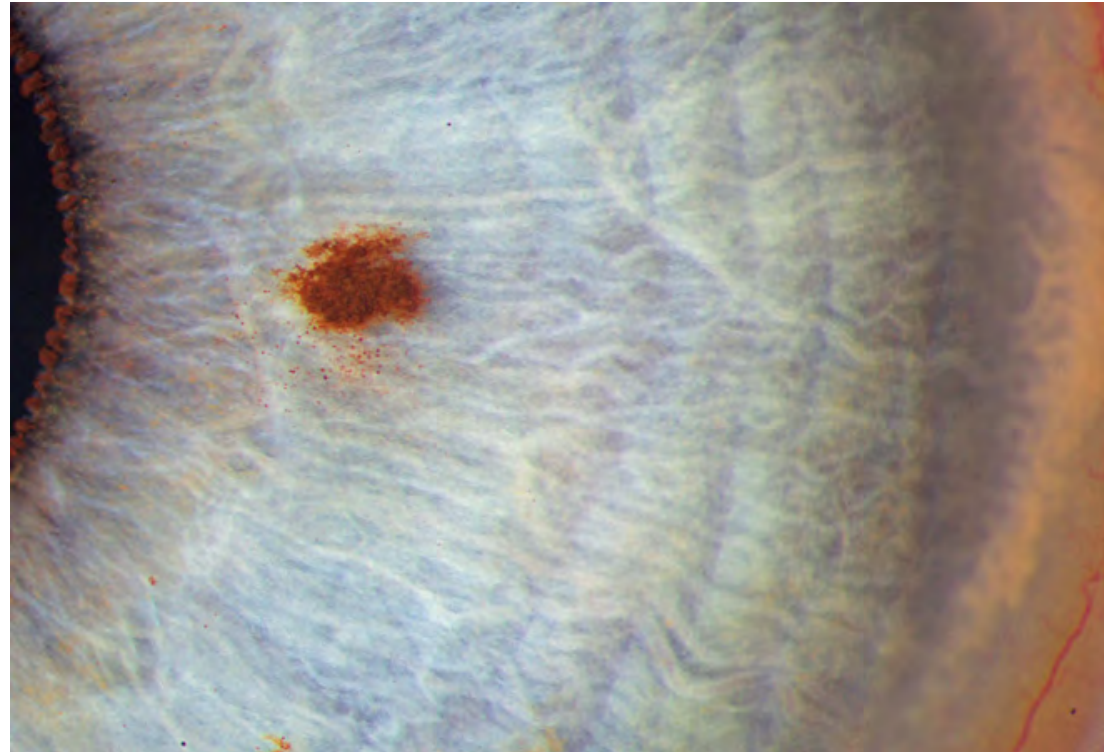

Abb. 6 Die Farbe eines Pigments erklärt das „Herkunftsorgan“. Die Lokalisation zeigt, wo es sich auswirkt. Ein Leberpigment im Herzfeld steht also für Hepatokardiales Syndrom.

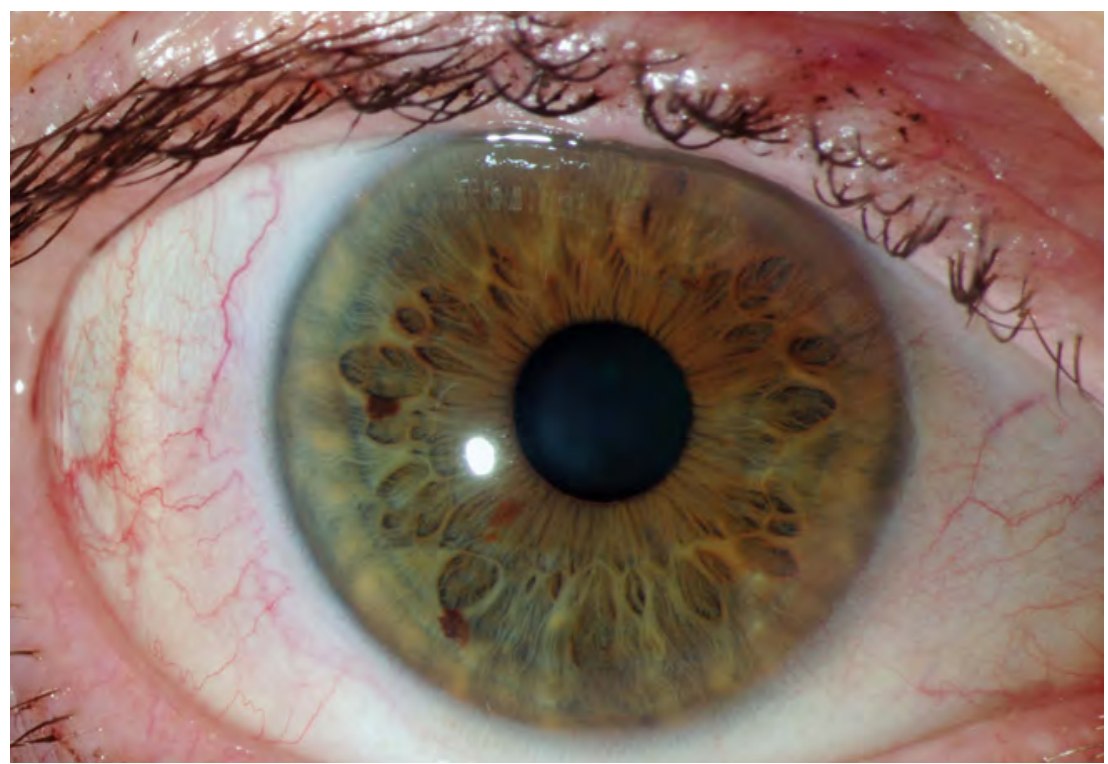

Abb. 7 Multipel auftretende Lakunen müssen systemisch bewertet werden. Klinisch diagnostizierbare endokrine Krankheiten finden sich eher selten.

triebsarmut und vegetativ-dystone $\mathrm{Zu}$ stände treten in diesem Zusammenhang auf.

\section{Irisfasern als Indikatoren neurogener Disposition}

Dünne, gespannte Irisfasern sind das Kennzeichen der neurogenen Disposition nach Deck. Auch die sogenannten „Neu- rohäkchen“, „erethische Kringel“ und das „Neuronennetz“ geben Aufschluss über Reizbarkeit und Schwächen des Nervensystems. Konstitution, Disposition, Diathese, Temperament, Sensibilität und Irritabilität geben nähere Hinweise auf die individuelle Reizaufnahme, -verarbeitung und -beantwortung. Im Rahmen einer pathogenetischen Reihe sind verschiedene Symptome möglich, darunter Sensibilitäts- und Irritabilitätserhöhun- 


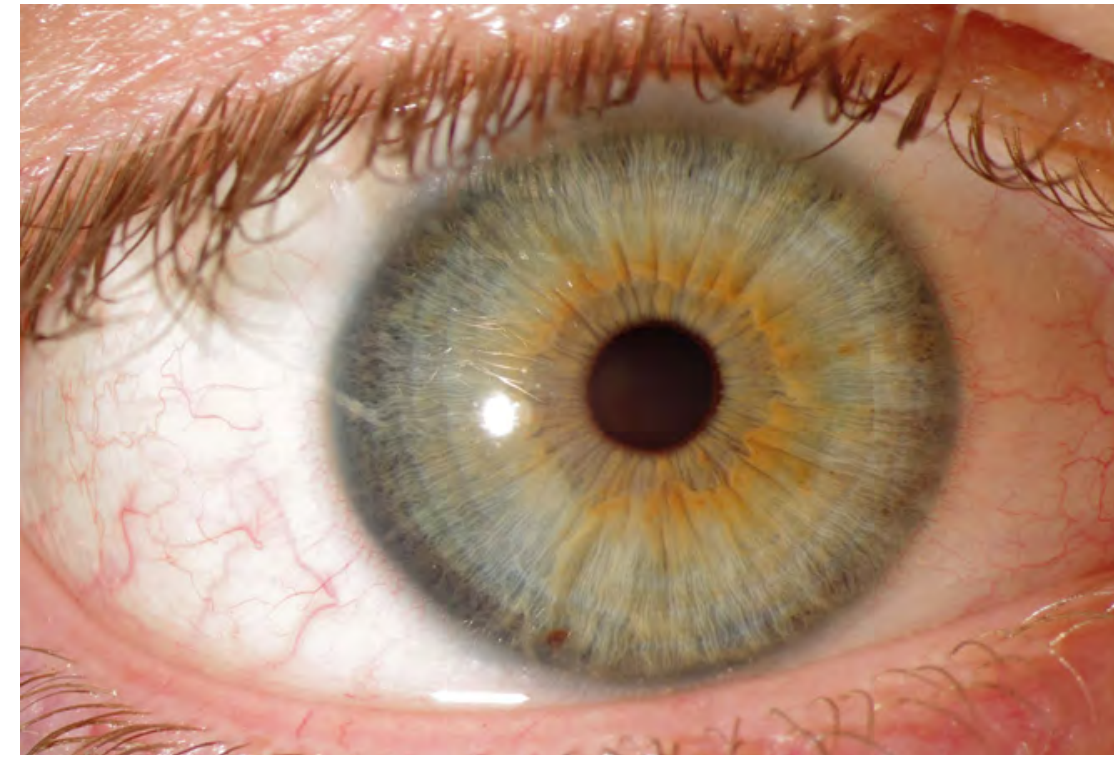

Abb. 8 In Zusammenhang mit der neurogenen Disposition findet man häufig psychosomatische Aspekte, die sich äußern können in Überforderungssyndromen, reizbarer Schwäche, Spasmen, Kopfschmerzen und Migräne.

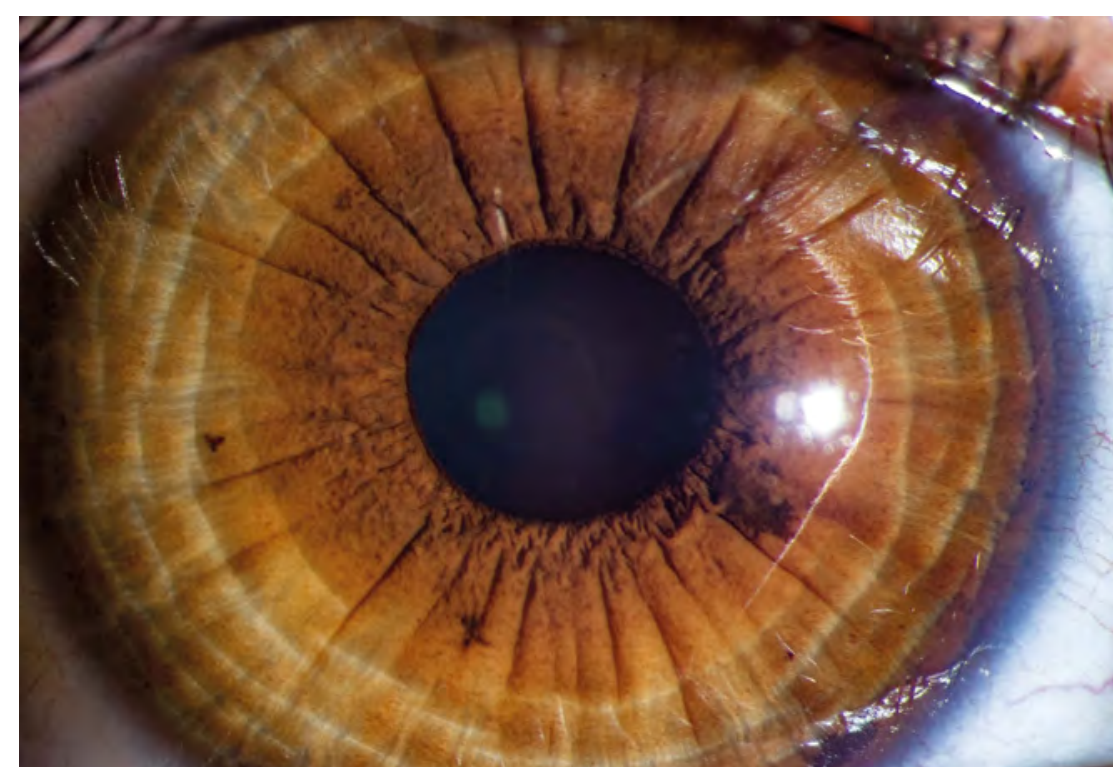

Abb. 9 Die Kombination von Zirkulär- und Radiärfurchen gilt als Kennzeichen einer vegetativ-spastischen Disposition.

gen, neurovegetative Störungen, Schmerzen, Katarrhe oder Entzündungen.

\section{Radiär- und Zirkulärfurchen: Hinweise auf zerebral-psychi- sche Einflüsse}

Jede Furchen- und Faltenbildung deutet auf eine konstitutionell gestörte Sympathikus-Parasympathikus-Balance hin. zum Beispiel Impulsivität wurde 2007 in einer schwedischen Studie bestätigt.

Verbindungslinien: erst bei Störungen sichtbar

Sichtbar werden Verbindungslinien erst, wenn entsprechende Funktionsstörungen in den jeweiligen Regelkreisen auftreten. Sie verbinden auf der Iris einander gegenüberliegende Organe und stellen so Bezüge und Abhängigkeiten dar. Das betrifft auch die Wechselbeziehung zwischen Emotionen und körperlichen Symptomen. Beispielsweise zeigt die horizontale Disharmonie-Linie eine Dysbalance zwischen oben und unten, Kopf (Psyche/Seele) und Körper (Soma). Im gleichen Sinn steht die senkrechte Gleichgewichtslinie für eine Dysbalance zwischen rechts und links beziehungsweise innen und außen. Schwindelzustände und Störungen des inneren Gleichgewichts sind dafür typisch.

\section{Fazit: Synergie von Befund, In- terpretation und Denkmodell}

Emotionsbedingte Organbelastungen entstehen auf vielfältige Weise. Die Iris ermöglicht nicht die klinische Diagnose, aber sie zeigt die konstitutionelle Grundlage, auf der diese Störungen entstehen und behandelt werden können. Die Augendiagnose geschieht hierbei in zwei Schritten: dem wahrnehmend-forschenden Blick mit dem Irismikroskop in das Auge und der diagnostischen Deutung. Dabei geht die Augendiagnose weit über eine rein klinische Diagnostik hinaus. Mit der Einbeziehung des naturheilkundlichen Denkmodells, auch im Hinblick auf die Kardinalsäfte und ihre Bedeutung für die Zonen- und Regionenlehre, betreten wir das Gebiet der Semiotik und Phänomenologie. Das bedeutet, dass wir unsere methodisch bedingten Erkenntnislücken durch Interpretation ersetzen müssen. Oder etwas profaner: Wir schließen von einem Modell auf die Wirklichkeit. Dies hat sich bewährt. Denn „der traditionellen Heilkunde (...) ist die strenge Trennung in Substanz - Substrat - Funktion fremd. Das gilt insbesondere für die matrizenähnliche 
Einteilung der Sachgebiete wie Anatomie, Physiologie usw. Darum sind auch von der Augendiagnose keine klinischen Diagnosen im letzteren Sinne zu erwarten. Der iridologische Befund ist eher eine Zustandsanalyse, aus der in die klinische Terminologie übersetzt werden muss.“ [3, S. 32]

Die Augendiagnose kann also (zumindest teilweise) erklären, wie und warum ein Mensch krank wird - eine wertvolle Hilfe für ein naturheilkundliches Therapiekonzept. Bewährt ist in diesem Zusammenhang das „Rezept aus dem Auge“ mit den Komplexmittelreihen zahlreicher biologischer Firmen. Aber die Augendiagnose fordert nicht zwingend die eine Therapieform ein, sondern eröffnet mannigfaltige und ganz individuelle Optionen.

Alle Abbildungen: ㄷ Hermann Biechele

Dieser Artikel ist online zu finden:

http://dx.doi.org/10.1055/s-0043-123323

\section{Literatur}

[1] Angerer J. Handbuch der Augendiagnostik. 5. Aufl. München: Tibor Marczell; 1984

[2] Biechele H. Basiswissen Augendiagnose. Ein Lehr- und Lernbuch. Kulmbach: ML; 2017

[3] Broy ]. Repertorium der Irisdiagnose. 3. Aufl. München: Klaus Foitzick; 2003

[4] Deck J. Grundlagen der Irisdiagnostik. Ettlingen: Selbstverlag des Verfassers 1965

[5] Lindemann G. Augendiagnostik Lehrbuch. Befunderhebung aus dem Auge. München: Tibor Marczell; 1984

[6] Schedlowski M, Tewes U (Hrsg.). Psychoneuroimmunologie. Heidelberg: Spektrum Akademischer Verlag; 1996

[7] Larsson M. (2007). Human Iris Characteristics as Biomarkers for Personality. Doctoral Dissertation. Faculty of Psychology. Publisher: Örebro University (www.oru.se/ub). Editor: Heinz Merten

[8] Schnabel R. Iridoskopie. Anleitung, Krankheiten und deren Veranlagung aus der menschlichen Iris zu erkennen. Ulm: Arkana; 1959

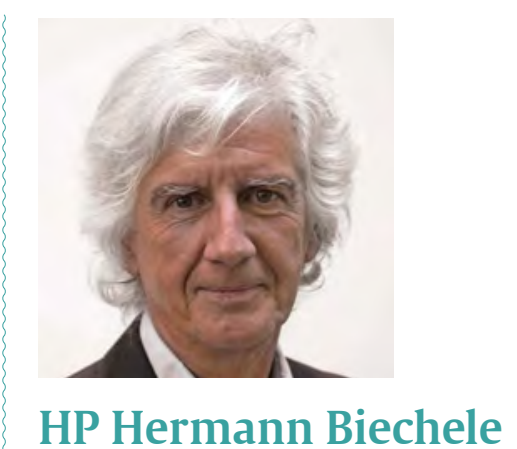

Kaiserstr. 51

80801 München

E-Mail: info@praxis-biechele.de

Hermann Biechele ist seit 1987 Heilpraktiker, erfahrener Dozent und Autor zahlreicher Veröffentlichungen zu verschiedenen Themen der Augendiagnose. Er leitet den „Arbeitskreis für Augendiagnose und Phänomenologie Josef Anger e. V." und unterrichtet das Fach Augendiagnose an der JosefAngerer-Schule in München. 